\title{
Número de repetições, de plantas por parcela e de avaliações para testes clonais de eucalipto
}

\author{
Márcio José de Araújo(1), Donizete da Costa Dias ${ }^{(2)}$, Edimar Aparecido Scarpinati( ${ }^{(3)}$ e Rinaldo Cesar de Paula ${ }^{(1)}$ \\ (1)Universidade Estadual Paulista Júlio de Mesquita Filho, Faculdade de Ciências Agrárias e Veterinárias, Via de Acesso Prof. Paulo Donato \\ Castellane, s/no, CEP 14884-900 Jaboticabal, SP, Brasil. E-mail: marcio ok66@hotmail.com, rcpaula@fcav.unesp.br (2)Fibria Celulose S.A., \\ Rodovia General Euryale de Jesus Zerbini, Km 84, São Silvestre, CEP 12340-010 Jacareí, SP, Brasil. E-mail: donizete.dias@fibria.com.br

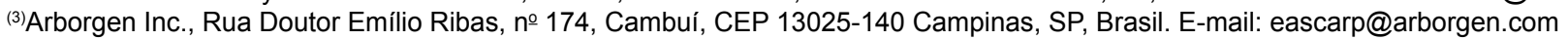

Resumo - O objetivo deste trabalho foi determinar o número de repetições, de plantas por parcela e de medições necessário para avaliação de caracteres de crescimento em testes clonais de eucalipto (Eucalyptus spp.). Foram avaliados dados de altura, diâmetro à altura do peito e volume de madeira, em três testes clonais, realizados em delineamento de blocos ao acaso, com 30 (testes 1 e 2) e 10 clones (teste 3), com seis repetições de seis (teste 1) e de 10 (testes 2 e 3) plantas. As avaliações foram realizadas dos 14 aos 74 meses de idade. Foram estimados os coeficientes de repetibilidade e de determinação, bem como os efeitos do acréscimo na frequência das medições sobre a eficiência seletiva. $\mathrm{O}$ volume de madeira é a característica com maior variabilidade na parcela e menor repetibilidade; portanto, exige maior número de plantas para que se obtenha elevada exatidão na estimativa do valor real do indivíduo. Em testes clonais de híbridos de Eucalyptus grandis x E. urophylla, recomenda-se o uso de três repetições, com seis plantas por parcela, e de três avaliações, em idades superiores a 2 anos, para que se alcance acurácia seletiva em torno de $90 \%$.

Termos para indexação: Eucalyptus, correlação intraclasse, eficiência seletiva, híbrido Urograndis, modelos mistos, repetibilidade.

\section{Number of replicates, of plants per plot and of evaluations for eucalyptus clonal tests}

\begin{abstract}
The objective of this work was to determine the necessary number of replicates, of plants per plot, and of measurements for evaluating growth traits in eucalyptus (Eucalyptus spp.) clonal tests. Data on height, diameter at breast height, and wood volume were evaluated in three clonal tests, performed in a randomized complete block design with 30 (tests 1 and 2) and 10 clones (test 3), with six replicates of six (test 1 ) and 10 (tests 2 and 3) plants. The evaluations were performed from 14 to 74 months of age. The coefficients of repeatability and determination were estimated, as well as the effects of increasing measurement frequency on selective efficiency. Wood volume is the trait with the greatest variability in the plot and the lowest repeatability; therefore, it requires more plants to obtain high accuracy in the estimate of the individual's true value. In Eucalyptus grandis x E. urophylla hybrid clonal tests, the use of three replicates of six plants per plot and of three evaluations, at ages above 2 years, is recommended in order to achieve a selective accuracy around $90 \%$.

Index terms: Eucalyptus, intraclass correlation, selection efficiency, Urograndis hybrid, mixed models, repeatability.
\end{abstract}

\section{Introdução}

Espécies do gênero Eucalyptus são as mais utilizadas em plantios de florestas comerciais no Brasil, em razão do seu rápido crescimento, da sua elevada produtividade, da sua ampla adaptação e da sua versatilidade de aplicação em diferentes usos. A área cultivada com eucalipto, portanto, tem crescido de forma sistemática ao longo dos anos, tendo atingido 5,47 milhões de hectares em 2013, 3,2\% superior à área cultivada em 2012 (Indústria Brasileira de Árvores, 2014).

O melhoramento florestal (Pinto Júnior et al., 2005), associado às técnicas silviculturais modernas (Xavier \& Silva, 2010), possibilita a formação de florestas mais eficientes e que atendam às características tecnológicas desejadas pelo mercado. Como o eucalipto é uma planta arbórea, de grande porte e de ciclo longo, os experimentos com a espécie demandam grandes áreas experimentais e dispêndios com recursos humanos e 
financeiros. Dessa forma, o uso de amostras que, além de representativas da população e do evento estudado, apresentem o menor tamanho possível, sem que haja perda das informações necessárias para o programa de melhoramento, é de grande relevância.

A fase final dos programas de melhoramento florestal envolve testes clonais, em que genótipos aprovados nos testes genéticos são propagados vegetativamente, em geral por miniestaquia, e colocados em competição em escalas piloto e até mesmo em plantios comerciais. Nesses testes, é importante que se determine o tamanho mínimo da amostra - que depende da combinação entre número de repetições e de plantas na parcela que garanta a confiabilidade dos dados obtidos. Essa informação é tão relevante quanto as estimativas da variabilidade genética e dos componentes de variância diversos, para a definição do método de seleção de indivíduos em uma população. Além disso, é comum que se realizem, nesses testes, avaliações periódicas do crescimento. A partir dessas avaliações, repetidas no tempo ou no espaço, pode-se estimar, por meio do coeficiente de repetibilidade, o número de medições necessário para uma eficiente seleção genotípica, o que reduziria custos e esforços despendidos no melhoramento (Cruz et al., 2012).

O coeficiente de repetibilidade vem sendo utilizado para determinar o número mínimo de avaliações, repetições e ambientes, entre outros aspectos, necessário para uma avaliação consistente de um determinado caráter ou de um genótipo. Exemplos com o uso desse parâmetro são frequentes em cultivos perenes ou semiperenes, como os de cana-de-açúcar (Ferreira et al., 2005; Cargnelutti Filho et al., 2012), eucalipto (Massaro, 2008), araçazeiro e pitangueira (Danner et al., 2010), capim-elefante (Cavalcante et al., 2012), pêssego (Bruna et al., 2012), pinhão-manso (Laviola et al., 2013), laranjeira-doce (Negreiros et al., 2008, 2014) e pinus (Cornacchia et al., 1995; Moreira et al., 2014). No entanto, ainda há poucas informações disponíveis na literatura para a cultura do eucalipto, como as disponibilizadas nos trabalhos de Resende (2002) e Massaro (2008), que mencionam estimativas de coeficientes de repetibilidade de média a alta magnitude, em geral superiores a 0,65 . Outro aspecto importante é que a seleção realizada entre 2 e 3 anos de idade tem apresentado alta correlação com a realizada aos 6 ou 7 anos, o que indica que a repetibilidade para caracteres de crescimento deve ser elevada (Massaro et al., 2010; Pinto et al., 2014).

O objetivo deste trabalho foi determinar o número de repetições, de plantas por parcela e de medições necessário para avaliação de caracteres de crescimento em testes clonais de eucalipto (Eucalyptus spp.).

\section{Material e Métodos}

Foram utilizados dados de três testes clonais com híbridos Eucalyptus grandis x E. urophylla, conduzidos no Município de Guatapará, SP $\left(21^{\circ} 30^{\prime} 0^{\prime \prime} \mathrm{S}, 47^{\circ} 59^{\prime} 0^{\prime \prime} \mathrm{W}\right)$. Utilizou-se o delineamento experimental de blocos ao acaso, com seis repetições e 30 tratamentos (clones), nos testes clonais 1 e 2 , e 10 tratamentos (clones) no teste 3. O eucalipto foi implantado com espaçamento $3,0 \times 3,0 \mathrm{~m}$ e parcelas lineares de seis plantas, para o teste clonal 1 , e de 10 plantas para os testes clonais 2 e 3 .

Foram avaliados o diâmetro à altura do peito $(\mathrm{cm})$ e a altura comercial (m). A partir desses caracteres, obteve-se o volume comercial das árvores, sem casca, $\left(\mathrm{m}^{3}\right.$ por árvore), pela aplicação dos fatores de forma e de casca, de acordo com a classe diamétrica. As avaliações foram realizadas nas idades de 25, 50 e 73 meses, para o teste clonal 1; de 26, 49 e 72 meses, para o teste clonal 2; e de 14, 20, 25, 51 e 74 meses, para o teste clonal 3.

As análises de variância foram realizadas separadamente para cada característica e teste clonal, tendo-se utilizado o arranjo de parcela subdividida para as idades de avaliação. Os dados foram analisados como modelos mistos, com uso do procedimento Proc Mixed do programa SAS, versão 9.3 (SAS Institute Inc., Cary, NC, EUA). Os componentes da variância para o cálculo do coeficiente de repetibilidade foram estimados com o procedimento da máxima verossimilhança restrita (REML). Utilizou-se o seguinte modelo estatístico, associado à avaliação de g genótipos em b blocos, com $\mathrm{n}$ indivíduos por parcela e $\mathrm{m}$ medições (idade) por indivíduo (Resende, 2002): $\mathrm{Y}_{\mathrm{ijk} \mathrm{l}}=\mu+\mathrm{g}_{\mathrm{i}}+\mathrm{b}_{\mathrm{j}}+\mathrm{e}_{\mathrm{ij}}+$ $\delta_{\mathrm{ijk}}+\mathrm{m}_{\mathrm{L}}+\mathrm{t}_{\mathrm{ijk} \mathrm{k}}$, em que $\mu$ é a média geral, $\mathrm{E}(\mu)=\mu \mathrm{e}$ $\mathrm{E}\left(\mu^{2}\right)=\mu^{2} ; \mathrm{g}_{\mathrm{i}}$ é o efeito aleatório do genótipo (clone), $\mathrm{E}\left(\mathrm{g}_{\mathrm{i}}\right)=0$ e $\mathrm{E}\left(\mathrm{g}_{\mathrm{i}}^{2}\right)=\sigma_{\mathrm{g}}^{2} ; b_{\mathrm{j}}$ é o efeito fixo do bloco $\mathrm{j}$, $\mathrm{E}\left(\mathrm{b}_{\mathrm{j}}\right)=\mathrm{b}_{\mathrm{j}}$ e $\mathrm{E}\left(\mathrm{b}_{\mathrm{j}}{ }_{\mathrm{j}}\right)=\mathrm{V}_{\mathrm{b}} ; \varepsilon_{\mathrm{ij}}$ é o efeito aleatório da parcela $\mathrm{ij}, \mathrm{E}\left(\mathrm{e}_{\mathrm{ij}}\right)=0$ e $\mathrm{E}\left(\mathrm{e}^{2}{ }_{\mathrm{ij}}\right)=\sigma_{\mathrm{e}}^{2} ; \delta_{\mathrm{ijk}}$ é o efeito aleatório do indivíduo $\mathrm{k}$ dentro da parcela $\mathrm{ij}, \mathrm{E}\left(\delta_{\mathrm{ijk}}\right)=0 \mathrm{e}$ $\mathrm{E}\left(\delta_{\mathrm{ijk}}^{2}\right)=\sigma_{\delta}^{2} ; \mathrm{m}_{\mathrm{L}}$ é o efeito fixo da medição $1, \mathrm{E}\left(\mathrm{m}_{1}\right)=0$ 
e E $\left(\mathrm{m}^{2}{ }_{\mathrm{l}}\right)=\mathrm{V}_{\mathrm{m}}$; e $\mathrm{t}_{\mathrm{ijkl}}$ é o efeito aleatório de ambiente temporário (erro), $\mathrm{E}\left(\mathrm{t}_{\mathrm{j} j \mathrm{kl}}\right)=0 \mathrm{e} \mathrm{E}\left(\mathrm{t}_{\mathrm{ijkl}}{ }_{\mathrm{j}}\right)=\sigma_{\mathrm{t}}^{2}$.

Os estimadores utilizados foram (Resende, 2002): repetibilidade nas avaliações (medições), $\hat{\rho}=\left(\widehat{\sigma}_{\mathrm{g}}^{2}+\bar{\sigma}_{\mathrm{e}}^{2}+\bar{\sigma}_{\delta}^{2}\right) /\left(\hat{\sigma}_{\mathrm{g}}^{2}+\bar{\sigma}_{\mathrm{e}}^{2}+\widehat{\sigma}_{\delta}^{2}+\hat{\sigma}_{\mathrm{t}}^{2}\right) ;$ repetibilidade de indivíduos na parcela, $\hat{\rho}_{\delta}=\widehat{\sigma}_{\delta}^{2} /\left(\hat{\sigma}_{\delta}^{2}+\widehat{\sigma}_{t}^{2}\right)$; e correlação intraclasse devida ao ambiente comum da parcela, $c^{2}=\widehat{\sigma}_{\mathrm{e}}^{2} / \widehat{\sigma}_{\mathrm{y}}^{2}$. Nessas equações, $\hat{\sigma}_{\mathrm{g}}^{2}$ é a variância genotípica; $\widehat{\sigma}_{\mathrm{e}}^{2}$ é a variância permanente entre parcelas (genótipo x bloco); $\hat{\sigma}_{\delta}^{2}$ é a variância permanente dentro de parcela; $\widehat{\sigma}_{t}^{2}$ é a variância de ambiente temporário; e $\widehat{\sigma}_{\mathrm{y}}^{2}$ é a variância fenotípica individual $\left(\widehat{\sigma}_{g}^{2}+\bar{\sigma}_{\mathrm{e}}^{2}+\bar{\sigma}_{\delta}^{2}+\bar{\sigma}_{t}^{2}\right)$.

O coeficiente de repetibilidade no bloco também foi estimado, ao nível de média de parcelas, por meio do seguinte modelo (Cruz et al., 2012): $\mathrm{Y}_{\mathrm{ij}}=\mu+\mathrm{g}_{\mathrm{i}}^{\prime}+$ $\varepsilon_{\mathrm{ij}}$, em que $\mathrm{Y}_{\mathrm{ij}}$ é a observação referente ao clone i na repetição $\mathrm{j} ; \mu$ é a média geral, $\mathrm{E}(\mu)=\mu$ e $\mathrm{E}\left(\mu^{2}\right)=\mu^{2}$; $\mathrm{g}_{\mathrm{i}}^{\prime}$ é o efeito aleatório do clone $\mathrm{i}$ sob a influência do ambiente permanente, $\mathrm{E}\left(\mathrm{g}_{\mathrm{i}}^{\prime}\right)=0$ e $\mathrm{E}\left(\mathrm{g}_{\mathrm{i}}^{\prime 2}\right)=\widehat{\sigma}_{\mathrm{g}}^{2}$; e $\varepsilon_{\mathrm{ij}}$ é o efeito do ambiente temporário associado à repetição $\mathrm{j}, \mathrm{E}\left(\varepsilon_{\mathrm{ij}}\right)=0$ e $\mathrm{E}\left(\varepsilon_{\mathrm{ij}}^{2}\right)=\sigma^{2}$.

O coeficiente de repetibilidade para repetições $\left(\widehat{\rho}_{\mathrm{b}}\right)$ foi calculado como:

$$
\hat{\rho} b=\frac{\operatorname{Cov}(Y i j, Y i j ')}{\sqrt{\widehat{V}(Y i j) \hat{V}\left(Y_{i j}\right)}}=\frac{\hat{\sigma}_{g}^{2}}{\sigma^{2}+\hat{\sigma}_{g}^{2}},
$$

em que $Y_{i j}$ e $Y_{i j}$ são as diferentes medidas realizadas em um mesmo indivíduo, nas diferentes repetições; e $\hat{\sigma}_{\mathrm{g}}^{2}$ e $\hat{\sigma}^{2}$ referem-se à variância genotípica (com ambiente permanente incluso) e de ambiente temporário, respectivamente.

As estimativas do coeficiente de determinação do valor real do indivíduo $\left(\mathrm{R}^{2}\right)$ e a eficiência seletiva por ciclo de seleção (EC) foram obtidas, de acordo com Resende (2002), pela equação:

$$
\mathrm{R}^{2}=\frac{\mathrm{n} \rho}{1+\rho(\mathrm{n}-1)} \text { e } \mathrm{EC}=\left[\frac{\mathrm{n}}{1+(\mathrm{n}-1) \rho}\right]^{0,5},
$$

em que $n$ é o número de medições realizadas e $\rho$ é o coeficiente de repetibilidade.

Para o teste clonal 3, foram realizadas duas análises: a primeira considerou as cinco idades de avaliação (14, 20, 25, 51 e 74 meses), denominada teste clonal 3a; e a segunda desconsiderou as idades de 14 e 21 meses, denominada teste clonal $3 \mathrm{~b}$. Este último foi realizado para comparação com os outros dois testes, em que foram feitas avaliações com idades semelhantes.

\section{Resultados e Discussão}

As estimativas da variância de clones na análise das plantas $\left(\widehat{\sigma}_{\mathrm{g}}^{2}\right)$, e da média de parcelas $\left(\widehat{\sigma}_{\mathrm{g}}^{2 \prime}\right)$ e de plantas dentro de parcelas $\left(\hat{\sigma}_{\delta}^{2}\right)$ foram significativas para os caracteres avaliados, nos três testes clonais (Tabelas 1 e 2). Isso indica que há variabilidade genética entre os clones, bem como efeito do ambiente dentro de parcelas.

Para as discussões, assumiu-se como desejável um coeficiente de determinação $\left(\mathrm{R}^{2}\right)$ de 0,81 , ou seja, uma acurácia seletiva de pelo menos $90 \%$, que, segundo Resende (2002) e Resende \& Duarte (2007), é um valor adequado para testes clonais de eucalipto e indica boa qualidade dos dados experimentais.

Ao se considerar as estimativas do coeficiente de repetibilidade (Tabela 3 ) e a eficiência seletiva por ciclo de seleção (Tabela 4), em termos do número de avaliações (medições), observa-se que o uso de mais de duas medições, nos testes clonais 1 e 2, e de quatro, no teste clonal 3, com cinco idades de avaliação (teste clonal 3a), acarretou pouco ganho na avaliação do diâmetro à altura do peito (DAP). Nos testes clonais 1 e 2, uma única medição foi suficiente para predizer o valor real do indivíduo, com determinação $\left(\mathrm{Ra}^{2}\right)$ de $81 \%$ (Figura 1), que corresponde a uma acurácia seletiva de 90\%. Esses resultados são consoantes com os obtidos por Cornacchia et al. (1995), para DAP em Pinus, que relataram repetibilidade de 0,76 , e por Massaro (2008), em eucalipto, que verificou repetibilidade de 0,82 . Nestes trabalhos, as medições 1 e 2 proporcionaram determinação de 80 e $90 \%$, respectivamente.

Para o caráter altura, constatou-se que não compensa realizar mais de quatro avaliações nos testes clonais $1\left(\mathrm{Ra}^{2}=0,86\right), 2$ e $3 \mathrm{~b}\left(\mathrm{Ra}^{2}=0,88\right.$ para ambos), para aumentar o ganho em acurácia no valor real do indivíduo (Tabela 4 e Figura 1). Três medições já foram suficientes para obter $\mathrm{Ra}^{2}$ de 0,81 ; contudo, no teste clonal $3 \mathrm{a}$, foram necessárias seis avaliações (Figura $1 \mathrm{G}, \mathrm{H}$ e I). Os resultados dos testes 1, 2 e 3b corroboram os de Massaro (2008), em um dos testes clonais de eucalipto avaliados, e os de Moreira et al. (2014), com Pinus elliottii var. elliottii.

As estimativas de repetibilidade individual para volume de madeira foram iguais às obtidas nos testes clonais 1 e $2(\hat{\rho}=0,597)$, e pouco inferiores à do teste clonal $3 b(\hat{\rho}=0,566)$. Essas estimativas proporcionaram estimativas de $\mathrm{Ra}^{2}$ próximas a $81 \%$ 
(Figura 1); para o teste clonal 3a, entretanto, esse número foi de oito, para um mesmo valor de $\mathrm{Ra}^{2}$. Resultados semelhantes foram relatados por Massaro (2008), em Eucalyptus spp., e estão próximos aos obtidos para Pinus (Cornacchia et al., 1995). Assim, para uma mesma exatidão no valor real do indivíduo, em experimentos clonais com eucalipto, é necessário maior número de medições do volume, quando comparado ao DAP e à altura. $\mathrm{O}$ volume possivelmente é controlado por diferentes conjuntos gênicos e fica sujeito a maiores variações e a menor estabilidade dos genótipos ao longo dos anos.

No teste clonal 3a, as estimativas de repetibilidade foram baixas, em comparação aos testes clonais 1 e 2 (Tabela 3); porém, ao se desconsiderar as avaliações de 14 e 21 meses de idade (teste clonal 3b), a repetibilidade assemelhou-se às dos testes clonais $1 \mathrm{e} 2$. Isso pode ter sido decorrente de conjuntos gênicos responsáveis por essas características, que não teriam se expressado ou que estariam menos ativos em idades mais jovens (Cruz et al., 2012). Ou seja, os genótipos apresentam maior correlação genética entre idades superiores a 2 anos do que entre idades mais jovens, o que está de acordo com o observado por Massaro et al. (2010), em Eucalyptus grandis. A competição menos intensa entre plantas nas idades precoces também pode ter contribuído para os menores coeficientes de repetibilidade obtidos com a inclusão de todas as avaliações na análise (teste clonal 3a), uma vez que os recursos do meio tornam-se mais escassos e a competição intensifica-se à medida que as plantas se desenvolvem.

Quanto à repetibilidade dos indivíduos na parcela $\left(\hat{\rho}_{\delta}\right)$, verificou-se que os ganhos em acurácia na avaliação do DAP deixaram de ser expressivos com mais de quatro plantas por parcela, nos testes 1 e $3 a$, e com mais de três plantas, nos testes 2 e $3 \mathrm{~b}$ (Tabela 4 e Figura 1). Em relação à altura, para obtenção de precisão de $81 \%$, o uso de mais de oito plantas, nos testes 1 e $3 \mathrm{a}$, e de seis plantas, nos testes 2 e $3 \mathrm{~b}$, não é vantajoso. Quanto ao volume, seriam necessárias pelo menos 10 plantas, no teste clonal 1, e 12 plantas, no teste clonal 3a, para alcançar $\mathrm{Ri}^{2}$ de $81 \%$. Para este mesmo valor de

Tabela 1. Estimativas dos componentes de variância genotípica $\left(\widehat{\sigma}_{\mathrm{g}}^{2}\right)$, variância de parcelas ( $\left.\widehat{\sigma}_{\mathrm{e}}^{2}\right)$ (clone vs. bloco), de planta dentro de parcela $\left(\widehat{\sigma}_{\delta}^{2}\right)$ [planta vs. (clone vs. bloco)] e de ambiente temporário $\left(\widehat{\sigma}_{t}^{2}\right)$, em três testes clonais ${ }^{(1)}$ com híbridos Eucalyptus grandis $\mathrm{x}$ E. urophylla, avaliados em diferentes idades quanto às variáveis diâmetro à altura do peito, altura das plantas e volume comercial sem casca.

\begin{tabular}{|c|c|c|c|c|c|c|c|c|c|c|c|c|c|c|c|c|}
\hline \multirow{2}{*}{$\begin{array}{l}\text { Componente } \\
\text { da variância }\end{array}$} & \multicolumn{4}{|c|}{ Teste clonal 1} & \multicolumn{4}{|c|}{ Teste clonal 2} & \multicolumn{4}{|c|}{ Teste clonal $3 \mathrm{a}$} & \multicolumn{4}{|c|}{ Teste clonal 3b } \\
\hline & lor & EP & $\mathrm{z}$ & $\mathrm{p}$ & olor & EP & $\mathrm{z}$ & $\mathrm{p}$ & Valor & EP & $\mathrm{z}$ & $\mathrm{p}$ & $\mathrm{r}$ & EP & $\mathrm{z}$ & $\mathrm{p}$ \\
\hline & \multicolumn{16}{|c|}{ Diâmetro à altura do peito $(\mathrm{cm})$} \\
\hline$\widehat{\sigma}_{g}^{2}$ & 2,2996 & 0,723 & 3,17 & 0,0008 & 1,9876 & 0,571 & 3,48 & 0,0003 & 0,8834 & 0,443 & 1,99 & 0,0231 & 1,5216 & 0,7713 & 1,97 & 0,0243 \\
\hline $\bar{\sigma}_{\mathrm{e}}^{2}$ & 2,1467 & 0,321 & 6,68 & $<0,0001$ & 0,7218 & 0,132 & 5,45 & 0,0001 & 0,0454 & 0,074 & 0,61 & 0,2696 & 0,1214 & 0,1497 & 0,81 & 0,2086 \\
\hline $\bar{\sigma}_{\delta}^{2}$ & 2,6206 & 0,160 & 16,29 & $<0,0001$ & 3,2438 & 0,135 & 24,04 & $<0,0001$ & 2,4391 & 0,178 & 13,69 & $<0,0001$ & 4,8116 & 0,3416 & 14,09 & $<0,0001$ \\
\hline \multirow[t]{2}{*}{$\hat{\sigma}_{t}^{2}$} & 1,9451 & 061 & 31,88 & $<0,0001$ & 1,2409 & 0,03 & 40,7 & $<0,0001$ & 2,2565 & 0,066 & 34,13 & $<0,0001$ & 2,0928 & 0,0872 & 24,01 & $<0,0001$ \\
\hline & \multicolumn{16}{|c|}{ Altura de plantas (m) } \\
\hline$\tilde{\sigma}_{g}^{2}$ & 2,1466 & 0,709 & 3,03 & 012 & 1,4008 & 0,387 & 3,62 & 0,0001 & 0,9023 & 0,476 & 1,9 & 0,0290 & 2,0061 & 1,0705 & 1,87 & 0,0305 \\
\hline $\bar{\sigma}_{\mathrm{e}}^{2}$ & 2,5838 & 382 & 6,76 & $<0,0001$ & 0,2496 & 0,052 & 4,81 & $<0,0001$ & 0,1567 & 0,137 & 1,15 & 00,126 & 0,4364 & 0,3342 & 1,31 & 0,0958 \\
\hline $\bar{\sigma}_{\delta}^{2}$ & 2,3498 & 0,196 & 11,98 & $<0,0001$ & 1,1956 & 0,066 & 18,18 & $<0,0001$ & 3,4673 & 0,294 & 11,8 & $<0,0001$ & 8,9094 & 0,6943 & 12,83 & $<0,0001$ \\
\hline \multirow[t]{2}{*}{$\hat{\sigma}_{t}^{2}$} & 4,4586 & 0,14 & 31,72 & $<0,0001$ & 1,5093 & 0,037 & 40,24 & $<0,0001$ & 6,1608 & 0,18 & 34,06 & $<, 0001$ & 6,0153 & 0,2529 & 23,79 & $<0,0001$ \\
\hline & \multicolumn{16}{|c|}{ Volume de madeira $\left(\mathrm{m}^{3}\right.$ por planta $)$} \\
\hline$\widehat{\sigma}_{g}^{2}$ & 0,0206 & 0,006 & 3,19 & 0007 & 0,0152 & 0,004 & 3,51 & 0,0002 & 0,0106 & 0,005 & 1,96 & 0,0249 & 0,0260 & 0,0133 & 1,95 & 0,0255 \\
\hline $\bar{\sigma}_{\mathrm{e}}^{2}$ & 0,0184 & 0,003 & 6,62 & $<0,0001$ & 0,0053 & 0,001 & 5,83 & $<0,0001$ & 0,0014 & 0,001 & 1,22 & 0,111 & 0,0040 & 0,0029 & 1,37 & 0,0859 \\
\hline $\bar{\sigma}_{\delta}^{2}$ & 0,0166 & 0,001 & 11,26 & $<0,0001$ & 0,0145 & 0,001 & 17,14 & $<0,0001$ & 0,0240 & 0,002 & 10,29 & $<0,0001$ & 0,0672 & 0,0058 & 11,60 & $<0,0001$ \\
\hline $\bar{\sigma}_{t}^{2}$ & 0,0375 & 0,001 & 31,93 & $<0,0001$ & 0,0237 & 0,001 & 40,77 & $<0,0001$ & 0,0668 & 0,002 & 34,15 & $<0,0001$ & 0,0745 & 0,0031 & 24,02 & $<0,0001$ \\
\hline
\end{tabular}

${ }^{(1)} \mathrm{O}$ teste clonal 3 foi realizado com cinco avaliações (3a), aos 14, 21, 25, 50 e 74 meses, ou com três avaliações (3b), aos 25, 50 e 74 meses. EP, erro-padrão. $\mathrm{p}$, significância estatística de acordo com o teste $\mathrm{z}$ de Wald. 
$\mathrm{Ri}^{2}$, sete e cinco plantas por parcela seriam suficientes para o teste clonal 2 e $3 \mathrm{~b}$, respectivamente. Contudo, o uso de mais de sete plantas acarreta em ganhos abaixo de $2 \%$ na eficiência seletiva (Tabela 4), para os testes clonais 1 e $3 \mathrm{a}$, e o mesmo ocorreria para o teste clonal 2 com o uso de mais de seis plantas.

Os testes clonais 2 e 3 apresentaram correlação intraclasse, pelo ambiente permanente comum na parcela $\left(\mathrm{C}^{2}\right)$, próxima a 0,10 (Tabela 3 ), dentro dos limites aceitáveis para bons experimentos e com pouca influência ambiental dentro das parcelas, para os três caracteres (Sturion \& Resende, 2005). Esses valores são consoantes com os relatados por Pinto et al. (2014), em testes clonais de Eucalyptus. No entanto, no teste clonal $3 \mathrm{a}$, dada as considerações citadas anteriormente, foram obtidas menores estimativas do coeficiente de repetibilidade, pelo fato de as avaliações dos genótipos terem sido feitas em idades inferiores a 2 anos. Quando foram desconsideradas essas idades (teste clonal $3 \mathrm{~b}$ ), os testes clonais 2 e 3 apresentaram estimativas próximas; sendo assim, a repetibilidade de indivíduos na parcela $\left(\hat{\rho}_{\delta}\right)$ provavelmente foi

Tabela 2. Estimativas dos componentes de variância genotípica influenciada pelo ambiente permanente $\left(\widehat{\sigma}_{\mathrm{g}}^{2 '}\right)$, em termos da média das parcelas e da variância de ambiente temporário $\left(\tilde{\sigma}_{\mathrm{t}}^{2}\right)$, em três testes clonais ${ }^{(1)}$ com híbridos Eucalyptus grandis $\mathrm{x}$ E. urophylla, avaliados em diferentes idades quanto às variáveis diâmetro à altura do peito, altura das plantas e volume comercial sem casca.

\begin{tabular}{|c|c|c|c|c|c|c|c|c|c|c|c|c|c|c|c|c|}
\hline \multirow{2}{*}{$\begin{array}{l}\text { Componente } \\
\text { da variância }\end{array}$} & \multicolumn{4}{|c|}{ Teste clonal 1} & \multicolumn{4}{|c|}{ Teste clonal 2} & \multicolumn{4}{|c|}{ Teste clonal $3 \mathrm{a}^{(1)}$} & \multicolumn{4}{|c|}{ Teste clonal $3 \mathrm{~b}^{(1)}$} \\
\hline & Valor & EP & $\mathrm{z}$ & $\mathrm{p}$ & Valor & EP & $\mathrm{z}$ & $\mathrm{p}$ & Valor & EP & $\mathrm{z}$ & $\mathrm{p}$ & Valor & EP & $\mathrm{z}$ & $\mathrm{p}$ \\
\hline & \multicolumn{16}{|c|}{ Diâmetro à altura do peito $(\mathrm{cm})$} \\
\hline$\hat{\sigma}_{\mathrm{g}}^{2^{\prime}}$ & 2,3491 & 0,740 & 3,18 & 0,0007 & 2,0448 & 0,5861 & 3,49 & 0,0002 & 0,902 & 0,4525 & 1,99 & 0,023 & 1,5304 & 0,778 & 1,97 & 0,0246 \\
\hline \multirow[t]{2}{*}{$\hat{\sigma}_{t}^{2}$} & 2,7610 & 0,324 & 8,51 & $<0,0001$ & 1,1133 & 0,1307 & 8,51 & $<0,0001$ & 0,345 & 0,0728 & 4,74 & $<0,0001$ & 0,7146 & 0,1507 & 4,74 & $<0,0001$ \\
\hline & \multicolumn{16}{|c|}{ Altura de plantas (m) } \\
\hline$\hat{\sigma}_{\mathrm{g}}^{2 \cdot}$ & 2,1672 & 0,713 & 3,04 & 0,0012 & 1,4215 & 0,3935 & 3,61 & 0,0002 & 0,9408 & 0,4979 & 1,89 & 0,0294 & 2,013 & 1,0738 & 1,87 & 0,0304 \\
\hline \multirow[t]{2}{*}{$\hat{\sigma}_{t}^{2}$} & 3,2203 & 0,378 & 8,51 & $<0,0001$ & 0,459 & 0,0539 & 8,51 & $<0,0001$ & 0,6846 & 0,1443 & 4,74 & $<0,0001$ & 1,5708 & 0,3312 & 4,74 & $<0,0001$ \\
\hline & \multicolumn{16}{|c|}{ Volume de madeira ( $\mathrm{m}^{3}$ por planta) } \\
\hline$\widehat{\sigma}_{\mathrm{g}}^{2^{\prime}}$ & 0,0206 & 0,006 & 3,190 & 0,001 & 0,0155 & 0,004 & 3,510 & 0,001 & 0,011 & 0,0056 & 1,96 & 0,0248 & 0,0267 & 0,0137 & 1,95 & 0,0256 \\
\hline$\underline{\hat{\sigma}_{t}^{2}}$ & 0,0237 & 0,003 & 8,510 & $<0,0001$ & 0,0078 & 0,001 & 8,510 & $<0,0001$ & 0,011 & 0,0056 & 1,96 & 0,0248 & 0,014 & 0,0030 & 4,74 & $<0,0001$ \\
\hline
\end{tabular}

${ }^{(1)} \mathrm{O}$ teste clonal 3 foi realizado com cinco avaliações (3a), aos 14, 21, 25, 50 e 74 meses, ou com três avaliações (3b), aos 25,50 e 74 meses. EP, erro-padrão. $\mathrm{p}$, significância estatística de acordo com o teste $\mathrm{z}$ de Wald.

Tabela 3. Estimativas dos coeficientes de repetibilidade para número de avaliações (anos, $\hat{\rho}$ ), número de plantas na parcela $\left(\widehat{\rho}_{\delta}\right)$ e número de repetições $\left(\widehat{\rho}_{\mathrm{b}}\right)$; dos coeficientes de determinação, em razão do número de avaliações $\left(\mathrm{Ra}^{2}\right)$, do número de plantas na parcela $\left(\mathrm{Ri}^{2}\right)$ e do número de repetições (blocos, $\left.\mathrm{Rb}^{2}\right)$; e do coeficiente de correlação devida ao ambiente comum da parcela $\left(\mathrm{C}^{2}\right)$, para os caracteres diâmetro à altura do peito (DAP, $\left.\mathrm{cm}\right)$, altura das plantas $(\mathrm{m})$ e volume comercial sem casca $\left(\mathrm{m}^{3}\right.$ por árvore) em três testes clonais ${ }^{(1)}$ com híbridos Eucalyptus grandis x E. urophylla, avaliados em diferentes idades.

\begin{tabular}{|c|c|c|c|c|c|c|c|c|c|c|c|c|}
\hline \multirow[t]{2}{*}{ Estimativas } & \multicolumn{3}{|c|}{ Teste clonal 1} & \multicolumn{3}{|c|}{ Teste clonal 2} & \multicolumn{3}{|c|}{ Teste clonal $3 \mathrm{a}^{(1)}$} & \multicolumn{3}{|c|}{ Teste clonal $3 \mathrm{~b}^{(1)}$} \\
\hline & DAP & Altura & Volume & DAP & Altura & Volume & DAP & Altura & Volume & DAP & Altura & Volume \\
\hline & \multicolumn{12}{|c|}{ Número de avaliações } \\
\hline$\hat{\rho}$ & 0,784 & 0,614 & 0,597 & 0,828 & 0,653 & 0,597 & 0,599 & 0,424 & 0,350 & 0,755 & 0,654 & 0,566 \\
\hline $\mathrm{Ra}^{2}$ & 0,916 & 0,827 & 0,816 & 0,935 & 0,850 & 0,816 & 0,882 & 0,786 & 0,729 & 0,902 & 0,850 & 0,797 \\
\hline \multirow[t]{2}{*}{$\mathrm{C}^{2}$} & 0,238 & 0,224 & 0,221 & 0,100 & 0,057 & 0,091 & 0,008 & 0,014 & 0,013 & 0,014 & 0,025 & 0,023 \\
\hline & \multicolumn{12}{|c|}{ Número de plantas na parcela } \\
\hline$\hat{\rho}_{\delta}$ & 0,574 & 0,345 & 0,307 & 0,723 & 0,442 & 0,380 & 0,519 & 0,360 & 0,264 & 0,697 & 0,597 & 0,474 \\
\hline \multirow[t]{2}{*}{$\mathrm{Ri}^{2}$} & 0,890 & 0,760 & 0,726 & 0,963 & 0,888 & 0,860 & 0,915 & 0,849 & 0,782 & 0,958 & 0,937 & 0,900 \\
\hline & \multicolumn{12}{|c|}{ Número de repetições } \\
\hline$\hat{\rho}_{\mathrm{b}}$ & 0,460 & 0,402 & 0,465 & 0,647 & 0,756 & 0,666 & 0,723 & 0,579 & 0,675 & 0,682 & 0,562 & 0,656 \\
\hline $\mathrm{Rb}^{2}$ & 0,836 & 0,802 & 0,839 & 0,917 & 0,949 & 0,923 & 0,940 & 0,892 & 0,926 & 0,928 & 0,885 & 0,920 \\
\hline
\end{tabular}

${ }^{(1)} \mathrm{O}$ teste clonal 3 foi realizado com cinco avaliações (3a), aos 14, 21, 25, 50 e 74 meses, ou com três avaliações (3b), aos 25,50 e 74 meses. 
subestimada na mesma proporção. Portanto, para uma boa reprodutibilidade de ensaios com Eucalyptus, as avaliações devem ser efetuadas em plantios com idade superior a 2 anos.

No teste clonal 1 , as estimativas de $\mathrm{C}^{2}$ foram maiores que as obtidas nos demais testes e superiores a 0,10 (Tabela 3), o que denota maior influência ambiental dentro das parcelas. Isso contribui para a expressão do caráter e reflete a necessidade de um número maior de indivíduos na parcela para aumentar a exatidão na seleção de genótipos.
O número adequado de plantas a ser utilizado nas parcelas variou de acordo com o caráter avaliado e seguiu, de forma inversa, as estimativas do coeficiente de repetibilidade: menor para o DAP, de maior repetibilidade; e maior para o volume, de menor repetibilidade. Com isso, testes clonais que visam selecionar genótipos de acordo com o volume de madeira demandam, pelo menos, seis plantas na parcela.

As diferenças observadas em relação aos resultados de Massaro (2008) podem ser atribuídas ao método de estimação dos componentes de variância, uma vez

Tabela 4. Eficiência seletiva pelo uso de m medições, em vez de uma medição, em testes clonais ${ }^{(1)}$ com híbridos Eucalyptus grandis $\mathrm{x}$ E. urophylla, avaliados em diferentes idades quanto aos caracteres diâmetro à altura do peito (DAP, $\mathrm{cm})$, altura das plantas $(\mathrm{m})$ e volume comercial sem casca ( $\mathrm{m}^{3}$ por árvore).

\begin{tabular}{|c|c|c|c|c|c|c|c|c|c|c|c|c|}
\hline \multirow[t]{2}{*}{$\mathrm{m}$} & \multicolumn{3}{|c|}{ Teste clonal 1} & \multicolumn{3}{|c|}{ Teste clonal 2} & \multicolumn{3}{|c|}{ Teste clonal 3a } & \multicolumn{3}{|c|}{ Teste clonal 3b } \\
\hline & DAP & Altura & Volume & DAP & Altura & Volume & DAP & Altura & Volume & DAP & Altura & Volume \\
\hline & \multicolumn{12}{|c|}{ Número de avaliações } \\
\hline 1 & 1,00 & 1,00 & 1,00 & 1,00 & 1,00 & 1,00 & 1,00 & 1,00 & 1,00 & 1,00 & 1,00 & 1,00 \\
\hline 2 & 1,06 & 1,11 & 1,12 & 1,05 & 1,10 & 1,12 & 1,12 & 1,19 & 1,22 & 1,07 & 1,10 & 1,13 \\
\hline 3 & 1,08 & 1,16 & 1,17 & 1,06 & 1,14 & 1,17 & 1,17 & 1,27 & 1,33 & 1,09 & 1,14 & 1,19 \\
\hline 4 & 1,09 & 1,19 & 1,20 & 1,07 & 1,16 & 1,20 & 1,20 & 1,33 & 1,40 & 1,11 & 1,16 & 1,22 \\
\hline 5 & 1,10 & 1,20 & 1,21 & 1,08 & 1,18 & 1,22 & 1,21 & 1,36 & 1,44 & 1,12 & 1,18 & 1,24 \\
\hline 6 & 1,10 & 1,21 & 1,23 & 1,08 & 1,19 & 1,23 & 1,23 & 1,39 & 1,48 & 1,12 & 1,19 & 1,25 \\
\hline 7 & 1,11 & 1,22 & 1,24 & 1,08 & 1,19 & 1,24 & 1,23 & 1,41 & 1,50 & 1,12 & 1,19 & 1,26 \\
\hline 8 & 1,11 & 1,23 & 1,24 & 1,09 & 1,20 & 1,24 & 1,24 & 1,42 & 1,52 & 1,13 & 1,20 & 1,27 \\
\hline 9 & 1,11 & 1,23 & 1,25 & 1,09 & 1,20 & 1,25 & 1,25 & 1,43 & 1,54 & 1,13 & 1,20 & 1,28 \\
\hline \multirow[t]{2}{*}{10} & 1,11 & 1,24 & 1,25 & 1,09 & 1,21 & 1,25 & 1,25 & 1,44 & 1,55 & 1,13 & 1,21 & 1,28 \\
\hline & \multicolumn{12}{|c|}{ Numero de plantas por parcela } \\
\hline 1 & 1,00 & 1,00 & 1,00 & 1,00 & 1,00 & 1,00 & 1,00 & 1,00 & 1,00 & 1,00 & 1,00 & 1,00 \\
\hline 2 & 1,13 & 1,22 & 1,24 & 1,08 & 1,18 & 1,20 & 1,15 & 1,21 & 1,26 & 1,09 & 1,12 & 1,16 \\
\hline 3 & 1,18 & 1,33 & 1,36 & 1,11 & 1,26 & 1,31 & 1,21 & 1,32 & 1,40 & 1,12 & 1,17 & 1,24 \\
\hline 4 & 1,21 & 1,40 & 1,44 & 1,12 & 1,31 & 1,37 & 1,25 & 1,39 & 1,49 & 1,14 & 1,20 & 1,28 \\
\hline 5 & 1,23 & 1,45 & 1,50 & 1,13 & 1,34 & 1,41 & 1,27 & 1,43 & 1,56 & 1,15 & 1,21 & 1,31 \\
\hline 6 & 1,25 & 1,48 & 1,54 & 1,14 & 1,37 & 1,44 & 1,29 & 1,46 & 1,61 & 1,16 & 1,23 & 1,33 \\
\hline 7 & 1,26 & 1,51 & 1,57 & 1,14 & 1,38 & 1,46 & 1,30 & 1,49 & 1,65 & 1,16 & 1,24 & 1,35 \\
\hline 8 & 1,26 & 1,53 & 1,59 & 1,15 & 1,40 & 1,48 & 1,31 & 1,51 & 1,68 & 1,17 & 1,24 & 1,36 \\
\hline 9 & 1,27 & 1,55 & 1,61 & 1,15 & 1,41 & 1,49 & 1,32 & 1,52 & 1,70 & 1,17 & 1,25 & 1,37 \\
\hline \multirow[t]{2}{*}{10} & 1,27 & 1,56 & 1,63 & 1,15 & 1,42 & 1,50 & 1,33 & 1,54 & 1,72 & 1,17 & 1,25 & 1,38 \\
\hline & \multicolumn{12}{|c|}{ Número de repetições } \\
\hline 1 & 1,00 & 1,00 & 1,00 & 1,00 & 1,00 & 1,00 & 1,00 & 1,00 & 1,00 & 1,00 & 1,00 & 1,00 \\
\hline 2 & 1,17 & 1,19 & 1,17 & 1,10 & 1,07 & 1,10 & 1,08 & 1,13 & 1,09 & 1,09 & 1,13 & 1,10 \\
\hline 3 & 1,25 & 1,29 & 1,25 & 1,14 & 1,09 & 1,13 & 1,11 & 1,18 & 1,13 & 1,13 & 1,19 & 1,14 \\
\hline 4 & 1,30 & 1,35 & 1,29 & 1,17 & 1,11 & 1,16 & 1,12 & 1,21 & 1,15 & 1,15 & 1,22 & 1,16 \\
\hline 5 & 1,33 & 1,38 & 1,32 & 1,18 & 1,11 & 1,17 & 1,13 & 1,23 & 1,16 & 1,16 & 1,24 & 1,17 \\
\hline 6 & 1,35 & 1,41 & 1,34 & 1,19 & 1,12 & 1,18 & 1,14 & 1,24 & 1,17 & 1,17 & 1,26 & 1,18 \\
\hline 7 & 1,36 & 1,43 & 1,36 & 1,20 & 1,12 & 1,18 & 1,15 & 1,25 & 1,18 & 1,17 & 1,27 & 1,19 \\
\hline 8 & 1,38 & 1,45 & 1,37 & 1,20 & 1,13 & 1,19 & 1,15 & 1,26 & 1,18 & 1,18 & 1,27 & 1,20 \\
\hline 9 & 1,39 & 1,46 & 1,38 & 1,21 & 1,13 & 1,19 & 1,15 & 1,26 & 1,19 & 1,18 & 1,28 & 1,20 \\
\hline 10 & 1,40 & 1,47 & 1,39 & 1,21 & 1,13 & 1,20 & 1,15 & 1,27 & 1,19 & 1,18 & 1,29 & 1,20 \\
\hline
\end{tabular}

${ }^{(1)} \mathrm{O}$ teste clonal 3 foi realizado com cinco avaliações (3a), aos 14, 21, 25, 50 e 74 meses, ou com três avaliações (3b), aos 25,50 e 74 meses. 

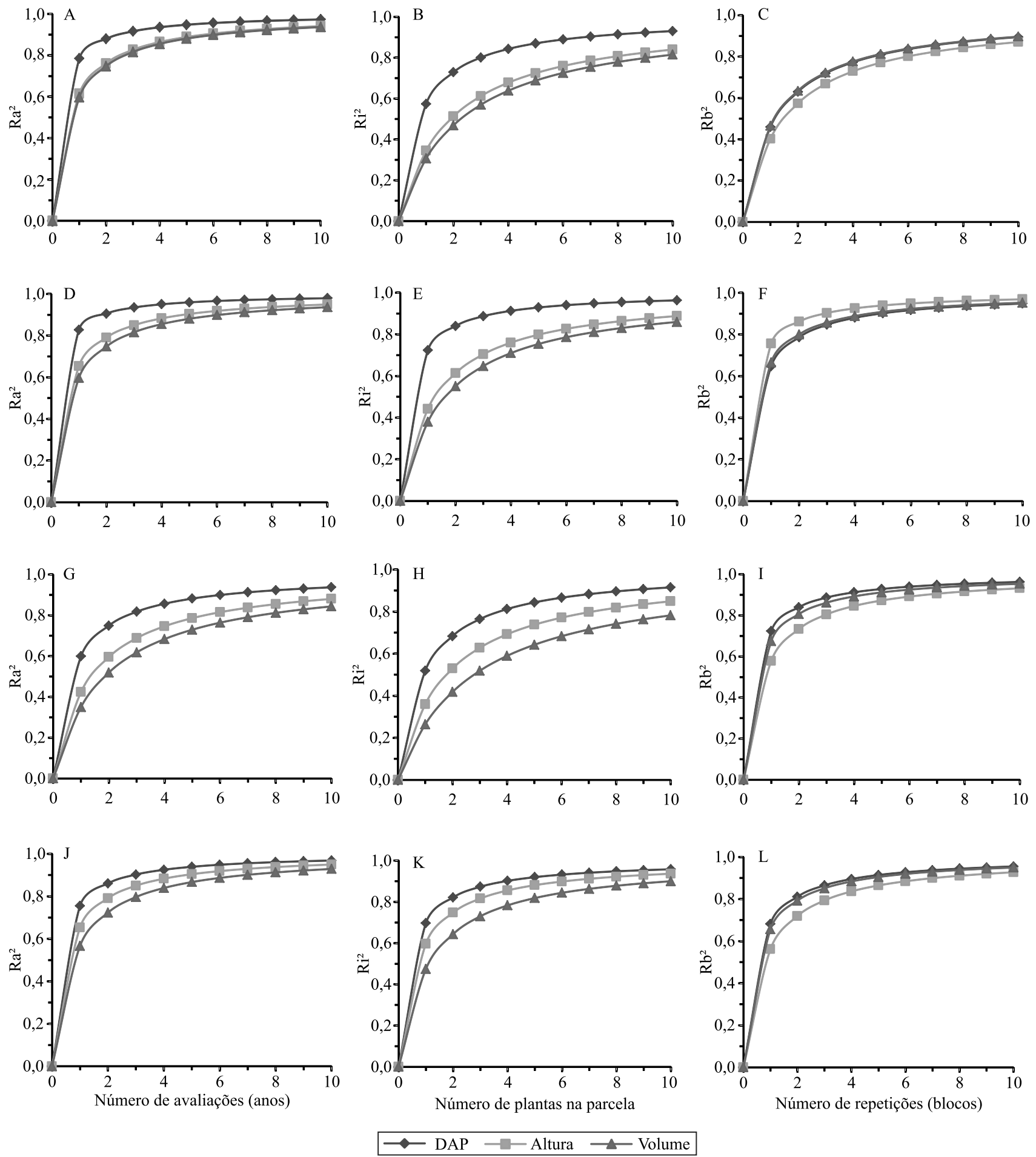

Figura 1. Estimativas do coeficiente de determinação do valor real do indivíduo em função do número de avaliações (anos, $\mathrm{Ra}^{2}$ ), do número de plantas na parcela $\left(\mathrm{Ri}^{2}\right)$ e do número de repetições (blocos, $\mathrm{Rb}^{2}$ ), com base nos coeficientes de repetibilidade, para os caracteres diâmetro à altura do peito (DAP), altura das plantas e volume comercial de madeira, referentes a três testes clonais com híbridos Eucalyptus grandis x E. urophylla, avaliados em diferentes idades: A, B e C, teste clonal 1 com idade de avaliação de 25, 50 e 73 meses; D, E e F, teste clonal 2 com idade de avaliação de 26, 49 e 72 meses; G, $\mathrm{H}$ e I, teste clonal 3a com cinco idades de avaliação, aos 14, 20, 25, 50 e 74 meses; e J, K e L, teste clonal 3b com três idades de avaliação, aos 25,50 e 74 meses. 
que, no presente trabalho, utilizou-se a metodologia REML, que considera os efeitos aleatórios do modelo estatístico associado às observações fenotípicas, e ajusta os dados aos efeitos fixos e ao número desigual de informações nas parcelas de acordo com a metodologia de modelos mistos (Henderson \& Quaas, 1976). Assim, os problemas de desbalanceamento nos dados, causados por falhas que resultam em parcelas com diferentes números de plantas, são devidamente corrigidos (Rocha et al., 2006).

No teste clonal 1 , cinco repetições para DAP e volume de madeira garantiram valores de $\mathrm{Rb}^{2}$ acima de $81 \%$ (Figura 1); já para altura, seis repetições proporcionaram estimativas de $\mathrm{Rb}^{2}$ de $80 \%$. Para os testes clonais 2 e 3 , três repetições foram suficientes para inferir quanto ao real valor do indivíduo com mais de $81 \%$ de determinação, para as três características.

No teste clonal 1, a influência do ambiente dentro das parcelas, detectada pelo coeficiente de correlação intraclasse devida ao ambiente permanente comum $\left(\mathrm{C}^{2}\right)$, refletiu a variabilidade espacial dentro de blocos (Sturion \& Resende, 2005). Daí se explica a baixa repetibilidade e a necessidade de um número maior de repetições para predizer o valor real do indivíduo com maior exatidão nesse teste. $\mathrm{O}$ teste clonal 2 foi o que apresentou maior concordância com o trabalho de Massaro (2008); porém, com estimativas de repetibilidade ligeiramente mais baixas.

\section{Conclusões}

1. Medições realizadas em testes clonais de eucalipto (Eucalyptus spp.) em idades inferiores a 2 anos subestimam o coeficiente de repetibilidade.

2. O volume de madeira está mais sujeito a expressar a variabilidade nas parcelas, o que resulta em menor repetibilidade de plantas na parcela e em maior número de plantas necessário para alcançar elevada exatidão no valor real do indivíduo.

3. Em testes clonais de híbridos de Eucalyptus grandis x E. urophylla, em que o objetivo da seleção é maximizar o ganho na geração atual para diâmetro à altura do peito, altura das plantas e volume de madeira, recomenda-se o uso de três repetições, com seis plantas por parcela, e de três avaliações, em idades superiores a 2 anos, para que se obtenha acurácia seletiva em torno de $90 \%$.

\section{Agradecimentos}

À Coordenação de Aperfeiçoamento de Pessoal de Nível Superior (Capes), pela concessão de bolsa de estudos ao primeiro autor; ao Conselho Nacional de Desenvolvimento Científico e Tecnológico (CNPq), pela concessão de bolsa de produtividade; e à Fibria Celulose S.A., pela disponibilização dos dados usados no trabalho.

\section{Referências}

BRUNA, E.D.; MORETO, A.L.; DALBÓ, M.A. Uso do coeficiente de repetibilidade na seleção de clones de pessegueiro para o litoral sul de Santa Catarina. Revista Brasileira de Fruticultura, v.34, p.206-215, 2012. DOI: 10.1590/ S0100-29452012000100028.

CARGNELUTTI FILHO, A.; BRAGA JUNIOR, R.D.C.; LÚCIO, A.D. Medidas de precisão experimental e número de repetições e ensaios de genótipos de cana-de-açúcar. Pesquisa Agropecuária Brasileira, v.47, p.1413-1421, 2012. DOI: 10.1590/ S0100-204X2012001000001.

CAVALCANTE, M.; LIRA, M. de A.; SANTOS, M.V.F. dos; PITA, E.B.A.F.; FERREIRA, R.L.C.; TABOSA, J.N. Coeficiente de repetibilidade e parâmetros genéticos em capim-elefante. Pesquisa Agropecuária Brasileira, v.47, p.569-575, 2012. DOI: 10.1590/S0100-204X2012000400013.

CORNACCHIA, G.; CRUZ, C.D.; PIRES, I.E. Estimativas do coeficiente de repetibilidade para características fenotípicas de procedências de Pinus tecunumanii (Schw.) Eguiluz \& Perry e Pinus caribaea var. hondurensis Barret \& Golfari. Revista Árvore, v.19, p.333-345, 1995.

CRUZ, C.D.; REGAZZI, A.J.; CARNEIRO, P.C.S. Modelos biométricos aplicados ao melhoramento genético. 4.ed. Viçosa: Ed. da UFV, 2012. v.1, 514p.

DANNER, M.A.; RASEIRA, M. do C.B.; SASSO, S.A.Z.; CITADIN, I.; SCARIOT, S. Repetibilidade de caracteres de fruto em araçazeiro e pitangueira. Ciência Rural, v.40, p.2086-2091, 2010. DOI: $10.1590 / \mathrm{S} 0103-84782010005000163$.

FERREIRA, A.; BARBOSA, M.H.P.; CRUZ, C.D.; HOFFMANN, H.P.; VIEIRA, M.A.S.; BASSINELLO, A.I.; SILVA, M.F. da. Repetibilidade e número de colheitas para seleção de clones de cana-de-açúcar. Pesquisa Agropecuária Brasileira, v.40, p.761-767, 2005. DOI: 10.1590/ S0100-204X2005000800005.

HENDERSON, C.R.; QUAAS, R.L. Multiple trait evaluation using relatives' records. Journal of Animal Science, v.43, p.1188-1197, 1976. DOI: $10.2134 /$ jas 1976.4361188x.

INDÚSTRIA BRASILEIRA DE ÁRVORES. A importância das árvores plantadas. Brasília, DF, [2014]. Disponível em: $<$ http:// www.iba.org/images/shared/folder-pt.pdf $>$. Acesso em: 25 nov. 2014. 
LAVIOLA, B.G.; OLIVEIRA, A.M.C. e; BHERING, L.L.; ALVES, A.A.; ROCHA, R.B.; GOMES, B.E.L.; CRUZ, C.D. Estimates of repeatability coefficients and selection gains in Jatropha indicate that higher cumulative genetic gains can be obtained by relaxing the degree of certainty in predicting the best families. Industrial Crops and Products, v.51, p.70-76, 2013. DOI: 10.1016/j. indcrop.2013.08.016.

MASSARO, R.A.M. Viabilidade de aplicação da seleção precoce e tamanho de parcelas em testes de clonais de Eucalyptus spp. 2008. 61p. Dissertação (Mestrado) - Faculdade de Ciênicas Agrárias e Veterinárias, Universidade Estadual Paulista "Júlio Mesquita Filho", Jaboticabal.

MASSARO, R.A.M.; BONINE, C.A.V.; SCARPINATI, E.A.; PAULA, R.C. de. Viabilidade de aplicação da seleção precoce em testes clonais de Eucalyptus spp. Ciência Florestal, v.20, p.597-609, 2010. DOI: 10.5902/198050982418.

MOREIRA, J.P.; SHIMIZU, J.Y.; SOUSA, V.A.; MORAES, M.L.T.; MOURA, N.F.; AGUIAR, A.V. de. Ganho esperado na seleção de progênies de Pinus elliottii var. elliottii em idade precoce para produção de madeira. Pesquisa Florestal Brasileira, v.34, p.99-109, 2014. DOI: 10.4336/2014.pfb.34.78.488.

NEGREIROS, J.R. da S.; ANDRADE-NETO, R. de C.; MIQUELONI, D.P.; LESSA, L.S. Estimativa de repetibilidade para caracteres de qualidade de frutos de laranjeira-doce. Pesquisa Agropecuária Brasileira, v.49, p.40-48, 2014. DOI: 10.1590/ S0100-204X2014000100006.

NEGREIROS, J.R. da S.; SARAIVA, L.L.; OLIVEIRA, T.K. de; ÁLVARES, V. de S.; RONCATTO, G. Estimativas de repetibilidade de caracteres de produção em laranjeiras-doces no Acre. Pesquisa
Agropecuária Brasileria, v.43, p.1763-1768, 2008. DOI: 10.1590/ S0100-204X2008001200017.

PINTO, D.S.; RESENDE, R.T.; MESQUITA, A.G.G.; ROSADO, A.M.; CRUZ, C.D. Seleção precoce para características de crescimento em testes clonais de Eucalyptus urophylla. Scientia Forestalis, v.42, p.251-257, 2014.

PINTO JÚNIOR, J.E.; STURION, J.A.; RESENDE, M.D.V. de; RONZELLI JÚNIOR, P. Efeitos da correção de dados na redução da heterogeneidade das variâncias genética, ambiental e fenotípica em testes de progênies de Eucalyptus grandis W. Hill ex Maiden. Pesquisa Florestal Brasileira, n.51, p.75-107, 2005.

RESENDE, M.D.V. de. Genética biométrica e estatística no melhoramento de plantas perenes. Brasília: Embrapa Informação Tecnológica; Colombo: Embrapa Florestas, 2002. 975p.

RESENDE, M.D.V. de; DUARTE, J.B. Precisão e controle de qualidade em experimentos de avaliação de cultivares. Pesquisa Agropecuária Tropical, v.37, p.182-194, 2007.

ROCHA, M. das G. de B.; PIRES, I.E.; ROCHA, R.B.; XAVIER, A.; CRUZ, C.D. Avaliação genética de progênies de meio-irmãos de Eucalyptus grandis por meio dos procedimentos REML/BLUP e da ANOVA. Scientia Forestalis, n.71, p.99-107, 2006.

STURION, J.A.; RESENDE, M.D.V. de. Eficiência do delineamento experimental e capacidade de teste no melhoramento genético da erva-mate (Ilex paraguariensis St. Hil.). Pesquisa Florestal Brasileira, n.50, p.3-10, 2005. DOI: 10.1590/ S0100-67622004000500005.

XAVIER, A.; SILVA, R.L. da. Evolução da silvicultura clonal de Eucalyptus no Brasil. Agronomía Costarricense, v.34, p.93-98, 2010.

Recebido em 15 de abril de 2015 e aprovado em 21 de julho de 2015 